\title{
Characteristic of Burst Transformations in Pseudoelasticity and Shape Memory Effect- a Review
}

\author{
K. Harikrishnan ${ }^{\mathrm{a} 1}$, P. S. Misra ${ }^{1}$, K. Chandra ${ }^{1}$, Vinod S. Agarwala ${ }^{2}$ \\ ${ }^{1}$ Metallurgical and Materials Engineering Department, IIT Roorkee, Roorkee-247 667, India. \\ ${ }^{2}$ Air Vehicle Engineering Department, US Naval Air Systems Command, Patuxent River, Maryland, USA.
}

\begin{abstract}
This paper reviews effect of the burst transformations in pseudoelasticity and shape memory effect in shape memory alloys (SMA). However, we will give some of our un-published results on tensile testing of Ti-49.83 at $\%$ Ni SMA wire sample which shows that burst transformations accompanying in-homogenous deformation of the stress-strain curve in pseudoelasticity region (above austenite finish temperature- $A_{f}$ ) proceeds to the upper yielding followed load drop similar to the upper and lower yield observed in the mild steel specimens. Though our stress-strain curve look similar to the one reported by P.Feng and Q.P.Sun, the stress before getting yield was $720 \mathrm{MPa}$ as compared to approx. $380 \mathrm{MPa}$ reported by them. An attempt was made to explain upper and lower yield point phenomenon with help of micro-mechanical modeling. Further research has to be directed to identify the relationship between microstructural changes with respect to the burst transformations and test temperature of the sample.
\end{abstract}

\section{Introduction}

\subsection{Historical background of shape memory alloys}

The first recorded observation of the shape memory transformation was by Chang and Read in $1932^{[1]}$. They noted the reversibility of the transformation in AuCd by metallographic observations and resistivity changes, and in 1951 the shape memory effect (SME) was observed in a bent bar of AuCd. In 1938, the transformation was seen in brass (copper-zinc). However, it was not until 1962, when Buehler and coworkers $^{[2]}$ discovered the effect in equiatomic nickeltitanium (Ni-Ti), that research into both the metallurgy and potential practical uses began in earnest. Memory alloys reported on the initial date are $\mathrm{Ti}-\mathrm{Nb}[3], \mathrm{Cu}-$ $\mathrm{Al}[4], \mathrm{Cu}-\mathrm{Al}-\mathrm{Ni}$ [5], Ni-Al [6], Cu-Zn [7], Fe-Pt [7], Fe-Ni [8], and $\mathrm{Cu}-\mathrm{Zn}-\mathrm{Sn}$ [9].

\subsection{Necessary conditions for the shape memory effect}

In order for the SME to operate, the following conditions must be satisfied [10]:

1. Stress induced martensite of either martensite or austenite (parent phase ) or reorientation of martensite [11].

2. These stress induced structural changes are stable after the removal of the external force.

3. A complete reversion to the original structure or substructure (including spatial orientation of the crystal or crystallites before the application of stress) upon heating or cooling through a transition temperature.

\footnotetext{
a e-mail: hari gce@yahoo.co.in
}

This is an Open Access article distributed under the terms of the Creative Commons Attribution-Noncommercial License (http://creativecommons.org/licenses/by-nc/3.0/), which permits unrestricted use, distribution, and reproduction in any noncommercial medium, provided the original work is properly cited. 
The shape memory effort, therefore, involves stress induced transformation and the degree of SME is related to the ease of reverse transformation. If reversible slip is involved either as the lattice invariant mode of deformation or due to stress accommodation then the ease of reverse transformation is reduced and the SME is greatly degenerated.

\subsection{Burst transformations}

The first reported martensitic transformation initiated by burst mode was probably by Machlin and Cohen [12] in $\mathrm{Fe}-\mathrm{Ni}$ and $\mathrm{Fe}-\mathrm{Ni}-\mathrm{C}$ alloys with sub-zero Ms temperature. Generalized statement for the burst transformations is "burst" occurs due to the autocatalytic nucleation of martensite suddenly from the austenite. These new nuclei grow such that they form into plates which accommodate the strains resulting from the formation of the first plate (which give rise to the autocatalytic nuclei), and a repetition of this basic process triggers the sudden zig-zag propagation of groups of mutually accommodating plates, resulted formation of burst transformations. The duration of the burst will be about $10^{-4}$ to $10^{-3}$ [13]. However, the burst usually stops before the transformation is complete, and this has been attributed to the exhaustion of nucleation sites. Burst transformations are found in many systems like $\mathrm{Au}-\mathrm{Cd}, \mathrm{Ni}-\mathrm{Ti}, \mathrm{Fe}-\mathrm{Ni}$ C alloys, Pu-Ga [14], etc.,

\subsection{Probability of distribution of Burst nuclei}

The distribution of Pre-existing nuclei spread have shown in equation (1) that the particles transform with probability $P$, such that a particle of volume $q$ contains at least one nucleation site $P=1-\exp \left(-q n_{v}{ }^{S}\right)$ where $\mathrm{n}_{\mathrm{v}}{ }_{\mathrm{S}}$ is the number of the said sites per-unit volume of material. That is, the sites for martensite nucleation would be randomly distributed in Fe-31.9Ni-0.02C alloys [15].

\subsection{Volume change (\%) and thermal hysteresis associated with the burst type transformations:}

Thermoelastic martensite crystal must be connected to the surrounding matrix crystal, and the coherency must be maintained during the whole process of the growth or shrinkage. In order to maintain coherency at the interface, the volume change associated with the transformation must be small in the first place. Infact, marmem associated with many marmem alloys are nearly $1 / 10$ of those for ferrous alloys or steel about (4\%) subjected to the burst type transformations, and thus the condition of the small volume change is satisfied, which is mentioned in Table-1.

Table-1 Relationship between volume change (\%) and thermal hysteresis in different shape memory systems.

\begin{tabular}{|c|c|c|c|}
\hline Alloy composition & Volume Change (\%) & Thermal hysteresis & References \\
\hline$(\mathrm{CuNi})_{3} \mathrm{Al}$ & -0.3 & 35 & 16 \\
$\mathrm{AuCd}$ & -0.41 & 15 & 17 \\
$\mathrm{In}-\mathrm{Tl}$ & -0.2 & 4 & 18 \\
$\mathrm{CuAuZn}$ & -0.25 & 6 & 19 \\
$\mathrm{CuZn}$ & -0.5 & 10 & 20 \\
$\mathrm{TiNi}$ & -0.34 & 30 & 21 \\
$\mathrm{NiAl}$ & -0.42 & 10 & 22 \\
$\mathrm{AgCd}$ & -0.16 & 15 & 23 \\
$\mathrm{Fe}_{3} \mathrm{Pt}$ & 1.1 & 4 & 24 \\
\hline
\end{tabular}

\subsection{Pseudoelasticity}

It is also possible to induce a phase transformation by applying a pure mechanical load isothermally at a temperature above austenite finish temperature. The result of this load application is fully detwinned martensite and very large strains are observed. The martensite formed in this way is known as stress- 
induced martensite and is only stable under the application of stress shown in Fig. 1. On unloading, the reduction in stress and surrounding elastic forces generated during transformation cause the martensite to shrink back to the original parent phase. This effect is known as pseudoelastic or superelastic effect $[11,25$ 26]

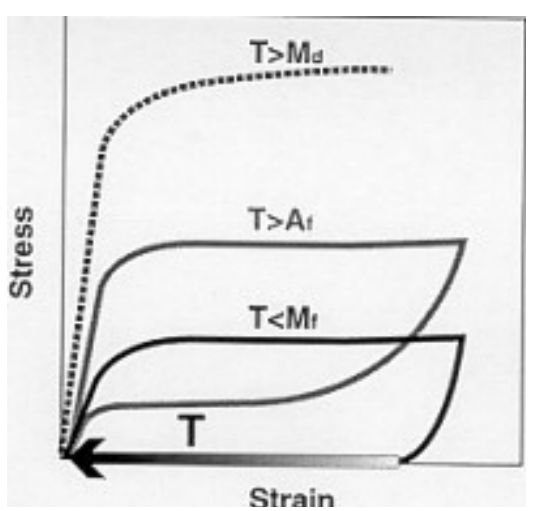

Fig. 1 stress-strain curve at different temperatures in shape memory alloys.

\subsection{Effect of temperature in Pseudoelasticity}

The stress necessary to create martensite transformation decreases with temperature being zero at Ms, the temperature at which martensite forms spontaneously on cooling. The variation $\sigma$ with temperature calculated from the clausius-clapeyron type equation applicable to SIM: $\mathrm{d} \sigma / \mathrm{dT}=\Delta \mathrm{H} /(\Delta \varepsilon$ To Vm) [27] where $\Delta \mathrm{H}$ is the heat of transformation, $\Delta \varepsilon$ the strain corresponding to the complete transformation to martensite, To the temperature at which the matrix and martensite phases are in equilibrium at zero stress and $\mathrm{Vm}$ is the molar volume.

\subsection{Thermoelastic and burst transformations}

The thermoelastic transformation is defined as one in which "a given plate or domain of martensite grows or shrink as the temperature is lowered or raised, and the growth rate appears to be governed only by the rate of change in temperature" [28-29] This definition implies fundamental distinction between the thermoelastic and other (burst? Isothermal?) types of transformation; therefore also the existence of at least to, and possibly more, different types of martensites also. The first reservations of this argument is experimental; it is quite straightforward to induce "burst" transformation in TiNi, in which between 50 and $80 \%$ of the specimen transforms apparently instantaneously with a loud click reminiscent of twin formation. Therefore thermoelastic behavior is not characteristics of $\mathrm{TiNi}$, a material exhibiting striking memory behavior.

The second reservation is more fundamental. It has become common over the years to describe differing experimental observations due to burst, isothermal, thermoelastic, etc., transformations. This usage may imply- or may lead to explicit assumptions of the existence of different kinds of martensite transformations. However, the fundamental nature of the martensite transformations remains regardless of the material, or of the condition under which the transformation takes place. Consequentially experimentally observed difference in behavior due to causes other than those directly and necessarily involved in the transformation itself. According to this hypothesis all the transformations are basically thermoelastic; therefore the shape memory effect should be inherently attainable in any material undergoing a martensite transformation. If so, it is only necessary to determine the conditions under which a complete shape memory occurs.

\subsection{Dislocations and burst martensites}

When cooled below Ms or stressed above Ms the first lamellae of early martensite form by dislocation lines which are most favorably oriented with respect to the driving force (chemical or mechanical); these can grow in to martensite plates when dislocations join the lamellae and dissociate. The dissociations can come 
from the network when they bow out between their nodes under an increasing driving force, screw dislocations may cross-slip, if not dissociated, and may multiply by the double cross-slip mechanism at the martensite plate interface [30-31].

When cooled below Ms the first thermoelastic martensite plates setup stress fields in regions between them due to the transformations dislocations at the edges of the lenticular plates with opposite stress fields and opposite shear in such away that there are no long range stress fields and no external shape strain develops. In contrast to this behavior, when the stress is applied to the untransformed parent above Ms the first lamellae will be thermoelastic, but these will form on plane, generally on single system, which have the greatest resolved shear stress; long range stress fields may develop in this case and stress induced thermoelastic martensite will show pseudoelastic behavior, with the lenticular plates shrinking and disappearing when the transformation dislocation return to their residual partials in the interference as the stress is removed, the driving force for this return being chemical [32]. If a partially transformed specimen is subsequentially deformed below Ms the applied stress will favor the growth of those plates for which the shear is the same, and the reversion of those for which the shear is opposite [33]. The applied stress effectively lowers the barrier to martensite formation in the regions between plates and both elastic and chemical driving forces tend to enhance formation of burst martensite when the resistive energy to initiate a martensite plate exceeds that for its propagation. Burst martensite will be enhanced by lower temperature below Ms and higher applied stresses, whereas thermoelastic martensite will form just below Ms, and under an applied stress at temperature above Ms; in the latter case the chemical driving force opposes stress induced martensite formation, and the tendency for burst martensite formation is reduced the higher the temperature is above Ms [34-36].

When the temperature is raised or the applied stress is removed the transformation dislocations reunite with their residuals, some of these perfect dislocations may move back to their previous network positions, but others may not, and those which formed at cross-slipping screw dislocations is likely. The finding that dislocation piles up suggesting that this may also be the shear plane for the transformations [37].

Since reversibility requires return of the transformation dislocation to their residuals, any obstacles or stress fields which limit this will introduce hysteresis into the stress-strain curve, and may reduce the amount of pseudoelasticity; the remaining shape strain which is reversible on heating constitutes the shape memory effect, and two effects pseudoelasticity and shape memory are complementary. Hysteresis develops when motion of the transformation dislocations is restricted, and is a measure of the relative energy available for the formation and reversion of the martensite plates; this differences approaches zero if all the plates are thermoelastic, and it may include part or all the deformation energy when the martensite plates are of burst type. When the burst martensite forms by stress below Ms and the stress is removed the restrictions present during the burst may be too large for complete return and a permanent strain sites in; this is recovered by heating when the shape memory effect applies.

Transformation dislocations which are tangled with other dislocations and with localized stress fields in burst martensite contribute to the hysteresis and loss of pseudoelasticity. When the plates extend to grain boundaries and transformation dislocations pass out during the burst there may be some loss in SME, in addition to the loss in pseudoelasticity, this depending on how effective the chemical driving force and back stress the residuals are in inducing transformation dislocations to move through steps in the grain boundaries. In this case pseudoelasticity and SME may not be complete complementary.

\section{Experimental studies on burst martensite}

The material used in the experiment was a polycrystalline NiTi wire with composition of $\mathrm{Ti}-49.8$ at $\% \mathrm{Ni}$ (NiTinol alloy supplied by US Navy, MD, USA). Using differential scanning calorimeter (DSC), the measured austenite finish temperature $\left(\mathrm{A}_{\mathrm{f}}\right)$ of the sample was $101^{\circ} \mathrm{C}$ so the material is in the austenite state at $125^{\circ} \mathrm{C}$ and will exhibit superelastic behavior under stress which was shown in Fig. 2 . The characteristic of the stress-strain curve linear up to $4.5 \%$ tensile straining was shown no changes in crystal structure and the sample having B2 phase only. However, beyond 4.5\% tensile strain, non-linear change in stress-strain path was happened due to the formation of stress-induced martensite and stress reaches maximum up to $620 \mathrm{MPa}$ with the total $9 \%$ strain. 


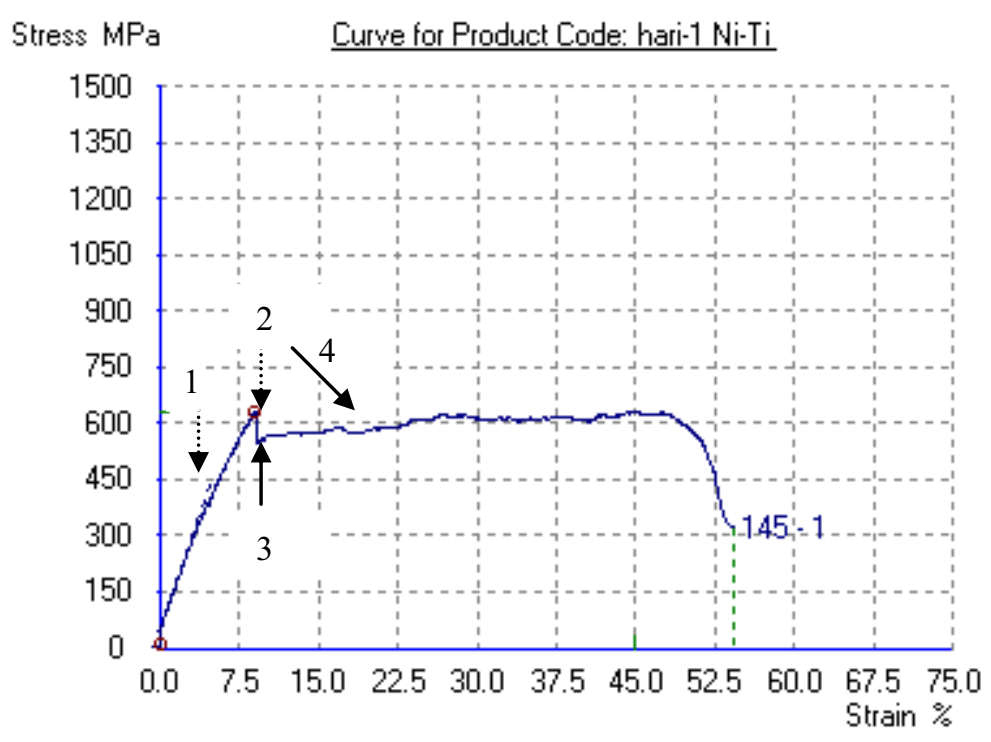

Fig. 2. Tensile stress-strain curve in NiTi shape memory alloy [38]

Thereafter, stress values drop promptly to $560 \mathrm{MPa}$ with constant strain. This sudden change in the stress state correspondingly due to the autocatalytic nucleation of the burst martensites. However, the metallographic studies over this wire sample not yet done. P. Feng and Q.P. Sun [39] also reportedly observed the same characteristics of the stress-strain curve and their $\mathrm{A}_{\mathrm{s}}$ temperature of the sample was $1{ }^{\circ} \mathrm{C}$ as against in this study was $101^{\circ} \mathrm{C}$.

Initially, the tensile test was done on the sample in austenitic condition which has $\mathrm{B} 2$ phase and the linear elongation was observed with respect to the linear increases in the applied tensile stress. However, the non-linear change in the deformation was observed once it reaches the peak load. Deformation at this stage could be divided into three sub-stages: (i) homogeneous elastic deformation of austenite; (ii) macroscopically homogeneous and stable transformation from austenite to martensite, referred to as " preburst transformation " or " incubation" and (iii) dynamic domain formation process which started at the peak load and was accompanied by a rapid load decrease in the S-S curve, as shown in Fig. 2.

(i) Macroscopically homogeneous transformation from B2-B19' martensite, referred to as "Pre-burst transformation" or "incubation": It's known that when the temperature increases above $\mathrm{A}_{\mathrm{f}}$ progressively high stresses are required to nucleate martensite and the curves are displaced to higher stress level with little change in the magnitude of the pseudo-elastic strain corresponding to the complete transformation to martensite. (ii) Where the gradual bending of the S-S curve with increasing deviation from linearity represented the $\mathrm{A} \leftrightarrow \mathrm{M}$ phase transition. To show such non-linearity is indeed due to phase transition. At sub-stage (iii), soon after the stress reached the peak value at point a (about $620 \mathrm{MPa}$ ), the deformation became inhomogeneous and uncontrollable through the dynamic formation (in the sense that it occurred very rapidly). A macroscopic deformation domain with a constant rapid nominal decrease of stress from $620 \mathrm{MPa}$ to $560 \mathrm{MPa}$ as shown in the Fig. 2, points 2 to 3 look similar to the upper yield and lower yield observed in mild steel.

The domain is actually the most transformed high strain region (martensite) of wire and was separated from the almost untransformed austenite (matrix) by a distinct domain boundary (or front region) across which the strain changed rapidly. After this the load reached a stable plateau value and the corresponding domain became a helical band with two spike-like tips. 


\subsection{Observation of Pseudoelasticity in tensile stress-strain curve}

A typical morphological change during the tensile test temperature greater than $A_{f}$ was shown in Fig. 2, along with the corresponding stress-strain curve. With increasing stress no morphological changes observed until the peak appears in the stress-strain curve. The peak point is a single variant of banded martensite appear and its interface between matrix and martensite propagate with extremely high velocity at this stress level. The rapid movement of the interface is associated with the rapid drop in stress as shown in the stressstrain curve. With increasing strain thereafter the stress level does not change greatly and the transformation proceeds by the propagation of both sides of interfaces of pre-existing martensite plates, no other martensites being nucleated in the untransformed area. This is the sharp in contrast described for the B2-B19' transformation by stress induced martensites. At point (4) the transformation within gauge length is almost completed and the stress again goes up rather rapidly. Thus, the strain between points 1 to $2(9 \%)$ represents the elongation associated with this stress-induced martensitic transformation. Even though, the stress-strain curve is similar to the pseudoelastic in nature as compared with work done by S. Miyazaki et al., (1981)[11]; the strain observed in the B2-phase region was very low about less than $2 \%$ before the formation of stress induced martensite will lead to the pseudo-elastic effect in their parent B2 lattice. However, in our present study, it is about $9 \%$ and the stress-strain curve is not so steep as compared with their experimental study. Therefore, it is clear that apart from super elasticity some microstructural changes are prone to the non-uniform deformation. Lars bergmans, et al., [40] also cited in their article in NiTi orthodontic wires, the phase changes along with the stress-induced transformation can be also thermoelastic or burst type of martensite.

\section{Discussion about the plausible reasons for existence of burst nucleation in the tensile sample based upon micro-mechanical model}

\subsection{Physical origin of the macroscopic deformation instability in polycrystals}

For the first type of instability (instability of the homogeneous deformation), it is seen from Fig. 2 that before the macroscopic domain nucleation the loading curve exhibited small nonlinearity due to the formation of many microscopic martensite bands in the polycrystal but the macroscopic deformation is still homogeneous. This homogeneous deformation (here termed as pre-burst incubation) persisted until the peak load that marks the beginning of macroscopic burst transformation. The pre-burst (macroscopically stable) and burst-like (autocatalytic) transformations (as already described in sub-stages (i) (iii) in detail) are typical NiTi polycrystals in tension $[41,42,43]$. The pre-burst was also observed in the transformation of other systems $[44,45,46,47]$. It was discussed in the literature using various terminologies such as autocatalytic effect, burst, avalanche, chain reaction, percolation, etc. There are two explanations for the possible physical mechanisms of the macroscopic deformation instability shown in Fig. 2. One speculated mechanism is the autocatalytic nucleation and coalescence of many micro-martensite bands or variants inside very small grains of the NiTi polycrystal. The autocatalytic transformation happened because the interaction among the local stress fields of these micro-domains formed in the pre-burst stage become increasingly strong with loading and eventually these micro-events percolate up to the macroscopic level and lead to the strain softening of a macroscopic representative material element until it is fully transformed. The phenomenon of autocatalytic, wherein a region of elastic phase transformation creates stresses sufficient to drive alone further transformations, was identified long time ago in the material systems mentioned above. In real polycrystals there is always a competition between the softening mechanism and the hardening mechanism (e.g. due to grain orientation) in the overall behavior. It is strongly influenced by the detailed microstructure (e.g. texture and grain size distribution) and the stress state (e.g. tension, shear or compression). In terms of elasticity, the softening stress-strain relation corresponds to a macroscopic non-convex strain energy function of the material during phase transition. Another possible mechanism leading to the macroscopic band formation, without invoking the intrinsic material softening, is the geometric instability due to the finite transformation strain. This is similar to the neck formation observed in some polymers where the material's true stress-strain relation is still stable. The 
investigation on this type of localized deformation has been well documented in the literature $[48,49,50$, 51]. Either geometric instability $[52,53,54]$ or material instability $[55,56]$ it is usually not straight forward from the measured nominal S-S curve to see immediately which one is dominant for the domain formation in the NiTi polycrystal. According to some micromechanical models [57,58] the elastic energy of a transforming polycrystal can be non-convex (softening stress-strain relation) for certain types of microstructures. One of the simplest form of non-convex energy is the Af(1-f) type, where A is a constant, $f$ is the volume fraction of a cluster of small spherical (circular in 2D) martensite variant for fine grained polycrystal. Another argument supporting the strain softening in tension is the measured value of load drop during macroscopic domain formation. It has been shown [59] that geometric instability alone (due to the finite $5 \%$ transformation strain of the domain) can only cause a relatively small load drop. The nonconvexity of strain energy function for the polycrystal is also supported by recent finite element simulation [60] on domain morphology in NiTi shape memory alloys. The results indicated that only geometry effect is not sufficient to quantify the experimental observation and that material instability dominates the observed helical domain formation. There have been notable theoretical efforts in recent years to model the nucleation peak, propagation plateau and hysteresis phenomena, emphasizing the discreteness and nonlocality of the phase transition process $[61,62,63]$. The microscopic instability developed at the macroscopic homogeneous deformation stage and continues after the macroscopic system becomes unstable. More detailed systematic measurement and quantitative modeling for real polycrystals along this line of thought remain to be conducted in the future.

\subsection{Mobility of the front and heat effect during domain pattern evolution}

In the present wire configuration a single helical domain could survive and grow after nucleation so that a nominal stress and front velocity relationship could be obtained. This measured relationship captured the macroscopic effect of those micro-thermomechanical events which facilitated the macroscopic front motion in polycrystals [64]. Usually a front kinetics expressed as $\mathrm{V}=\mathrm{V}(\mathrm{F})$, relating the front velocity $\mathrm{V}$ and the thermodynamic driving force $\mathrm{F}$ at the front, is supposed to exist and is proposed as an intrinsic constitutive function of the material. It is known that the latent heat generated as the front propagates during $\mathrm{A} \leftrightarrow \mathrm{M}$ transition will reduce the chemical driving force, so the front velocity is limited by heat transfer. At the same time, as the domain grows, the resulted load drop will also reduce the mechanical driving force of the front, accompanied by a rapid decrease in front velocity. In a one-dimensional setting, such thermomechanical coupling in the measured nominal kinetic relationship has been analyzed by several researchers (see Shield et al., [65] and Shaw [66]). In their work, by considering the steady state motion of a single interface in the wire and taking into account the latent heat generated during front propagation, a closed-form relationship between the steady -state interfacial speed and the overstress (i.e. the difference between the applied stress and the plateau stress) was derived (Bruno et al.,[67] and Shield et al.,[68]). Research on the macroscopic front kinetics of polycrystalline NiTi [69] and Cu-Al-Ni single crystals [61] demonstrated that the kinetic function has some common characteristics with those of frictional behavior in that a small change in the driving force will lead to a rapid change in front velocity. Because of the strong similarity between dislocation and displacive transformation ("martensitic") at the microscopic scale, there are strong parallels between the kinetics of displacive transformation and plastic deformation [70].

This means that for isothermal quasistatic front motion, the total driving force (i.e. Maxwell stress plus the dissipative force (in loading)) is independent of the front speed, or the speed of the front is exactly what is required for the front to have a constant driving force. Thus for the quasistatic motion of a macroscopic front obtained from the intrinsic macroscopic front kinetics $F=F(V)$ could be treated as the rateindependent type or perfect-plasticity type. The measured apparent stress-dependency of the front velocity is mainly a manifestation of the thermal effect which is masked onto the intrinsic isothermal kinetics. As the summary of this section, the roles of energetics and kinetics as well as their interplay in governing the observed domain evolution are schematically plays systematically vital role on prompt load drop observed in tensile specimen. 


\title{
4. Conclusions
}

The characteristics of the burst transformation with respect to the thermoelastic martensites, dislocation, nucleation and distribution probability, pseudoelasticity are reviewed in short but elaborately. The authors also observed burst transformations with pseudoelastic region with temperature greater than austenitic finish temperature. The burst transformation associated with tensile stress-strain curve was found in NiTi samples look similar to the upper and lower yield point in mild steel specimens. However, in this present study in-homogenity in tensile stress-strain curve high elongation (nearly $9 \%$ strain) is required before proceeds to the load drop. The total pseudoelastic strain observed in this specimen was very high $47.5 \%$. Hence, further research has to be directed to identify the relationship between microstructural changes with respect to the burst transformations and test temperature of the sample.

\author{
Acknowledgements \\ The author K. Harikrishnan expresses his sincere thanks to the staff at Instrument Instrumentation Center in IIT \\ Roorkee, who helped them lot to carry out the DSC and In-situ XRD studies and his sincere gratitude to Naval Air \\ Systems Command, United States Navy who provided NiTi shape memory alloys for his M.Tech dissertation work.
}

\section{References}

[1] L.C. Chang and T.A Read, Trans. AIME, Vol. 191, (1951), p 47

[2] W.J. Buehler, J.V. Gilfrich, and R.C. Wiley, J. Appl. Phys., Vol.34, (1963), p 1475

[3] C. Baker, Metal Sci. Journal,. Vol.5, (1971), p. 92.

[4] Nagasawa and K. Kawachi, J. Phys. Soc. Japan., Vol. 30, (1971), p. 296

[5] K. Otsuka and K. Shimizu, Scripts Met. Vol.4, (1970), p. 469

[6] K. Enami and S. Nenno, Met. Trans., Vol.2, (1971), p. 1487

[7] C.M. Wayman, Scripts Met. Vol.5, (1971), p. 487.

[8] Nagasawa, J. Phys. Soc. Japan, Vol.30, (1971), p.1505.

[9] J.D. Eisenwasser and L.C. Brown, Met. Trans.,3, 1359 (1972)

[10] Jeff Perkins, Ed. Shape memory effects in alloys, Plenum Press, Newyork (1975).

[11] S. Miyazaki, K. Otsuka, Y. Suzuki, Scripta Metall., Vol.15, (1981), p. 287

[12]E. S. Machlin and M. Cohen, Trans. AIME, Vol.191,(1951), p.746.

[13] J.R.C. Guimaraes Materials Science and Engineering, Vol.95, (1987), p. 217-224

[14] J.N. Mitchell, F.J. Freibert, D.S. Schwartz, M.E. Bange, Journal of Nuclear Materials, Vol. 385 (2009), p. $95-98$

[15] J. R. C. Guimar£es and A. Saavedra, Mater. Sci.Eng., Vol.62, (1984), p. 11.

[16] C.M. Wayman, I. Cornelis, and K. shimizu, Scripta Met., Vol.6, (1974) ,p.1524

[17]Z. Zirinsky, Acta Met., Vol.5, (1971), p. 127

[18] L. Guttman, Trans. AIME, Vol.188,(1950), p. 1472

[19] N. Nakanishi, Y. Murakami and S. Kachi, J. Appli Phyiscs, Vol.11, (1972), p. 1591

[20] A.L. Titchener, and M.B. Bever, Transaction AIME, Vol.198, (1954), p.303

[21] K. Ostuka and K. Shimizu, Scripta Met., Vol.4, (1970), p.469.

[22] Y.K. Au and C.M. Wayman, Scripta Met., Vol.6,(1972), p. 1209

[23] D. M. Masson and C.S. Barrett, Trans., AMIE, Vol.212, (1958), p.260.

[24] T. Tadaki and K. Shimizu, Trans. Inst Met., Vol.14, (1973), p. 161.

[25] Worden K. Bullough W. A., Haywood J., Smart technologies, World Scientific (2003), pp.109-135

[26] K. Otsuka, K. Shimizu, Int Metals Rev., Vol.31, (1986), p.93.

[27] M.W. Burkart, T.A. Read, Trans AIME, Vol.197, (1953), p.1516

[28] GD Sandrock, R.F. Hehemann, Metallography, Vol.4, (451), p.1971.0,

[29] K. Otsuka, T. Sawamura , K. Shimizu, C M. Wayman. Metall Trans., Vol.2, (1971), p.2583.

[30] C.M.Wayman, and K. Shimizu, Met. Sci. J, Vol.6, (1972), p. 175;

[31] L. Delaey, and R.V. Krishnan, H. Tas, and H. Warlimint, I, II, and III, J. Mat. Sci., Vol.9, (1974), p. $1521,1536,1545$

[32] G B Olso, M. Cohen., Scripta Metall., Vol.9, (1975), p. 1247 
[33] S. Ii , K. Yamauchi, Y. Maruhashi, M. Nishida, Scripta Mater., Vol.49, ( 2003), p.723

[34] J. D. Eisenwesser and L C Brown, Met Trans., Vol.3, (1972), p.1359.

[35] K. Oshi and L C Brown, Met. Trans., Vol.4, (1971), p.1971

[36] R. V. Krishnan and L.C. Brown, Met. Trans., Vol.4, (1973), p.423

[37] J. Perkins, Met. Trans., Vol.4, (1973), p.2709

[38]K. Harikrishnan, Characterizations of NiTi Shape memory systems, M.Tech disseratation report (2005), IIT Roorkee, Roorkee, India.

[39]P. Feng and Q.P. Sun, Journal of the Mechanics and Physics of Solids, Vol.54, (2006), p. 1568-1603

[40] Lars Bergmans, Johan Van Clfynenbreugel, Martine Wevers, \& Paul Lambrecchts, American Journal of Dentistry, Vol. 14, No. 5, October, (2001).

[41]Z.Q. Li, and Q.P. Sun, Int. J. Plasticity Vol.18 , (2002), p. 1481-1498.

[42] Q.P Sun, and Z.Q. Li, Int. J. Solids Struct ., Vol. 39 , (2002), p.3797-3809.

[43] Q.P Sun, Z.Q. Li, and K. K Tse, Proceedings of IUT AM symposium on smart structures and structronic systems, U. Gabbert and H. S. Tzou (eds.), Kluwer Academic Publishers, (2001) p, 113 120.

[44]Z. Nishiyama, Martensitic Transformation, Academic Press, New York, (1978)

[45]P. E. Reyes-Morel, and I.W. Chen, J. Am. Ceram. Soc., Vol. 72, (1988), p. 343-353.

[46] L. R. F. Rose, and M.V. Swain, Acta Metall., Vol.36 , (1988), p.955-962.

[47]Z.Z. Y u, and P.C. Clapp, Metal. T rans. A 20 , (1989) p. 1601-1615.

[48] Needleman, and V. Tvergaard, Appl. Mech. Rev. 45 , (1992), S3-S18.

[49] Q.S. Nguyen, Appl. Mech. Rev. 47 , (1994), p.1-31.

[50] Y. Tomita, Appl. Mech. Rev . 47 , (1994), p.171-205.

[51] H. Petryk, Arch. Comp. Mech. Eng., Vol. 4 , (1997) ,p.111-151.

[52]L. Orgeas, and D. Favier, Stress-induced martensitic transformation of a NiTi alloy in isothermal shear, tension and compression. Acta Mater., Vol. 46, (1998), p.5579-5591.

[53] D. Favier, Y. Liu, L. Orgeas and R. Rio Solid Mechanics and Its Applications, Vol. 101, ed. By Q. P . Sun, Kluwer Academic Publisher, (2001), p.205-212.

[54] Sittner, Y. Liu, and V. Novak, J. Mech. Phys. Solids, Vol.53 , (2005), p.1719-1746.

[55]F. Falk, Z. Physik B - Condensed Matter, Vol.51 , (1983), p.177-185.

[56] G.R. Barsch, and J.A. Krumhansl, Metallurg. Trans., A 19 , (1988), p.761-775.

[57] Q.P Sun, K.C. Hwang, and S.W, Y u, J. Mech. Phys. Solids, Vol. 39 , (1991), p. 507-524.

[58] O.P Bruno, P. H. Leo, and F. Reitich, Phys. Rev. Lett. 74, (1995), p.746-749.

[59] J. A. Shaw, and S. Kyriakides, Int. J. Plasticity, Vol.13 , (1998), p.837-871

[60] Y. J. He, and Q. P. Sun, Proc. of Int. Conf. on Solid-Solid Phase Transformations in InoganicInorganic Mater ., May 29-June 3, Phoenix, Arizona, USA (2005)

[61] Abeyaratne, C. Chu, and R.D. James, Phil. Mag. A 73 , (1996), p. 457-497.

[62] J. Ortin and L. Delaey, Int. J. Nonlinear Mechanics, Vol.37 , (2002), p.1275-1281.

[63] N. Triantafyllidis, and S. Bardenhagen, J. Elasticity, Vol. 33 , (1993), p.259-293.

[64] R. Abeyaratne, and J.K. Knowles, J. Mech. Phys. Solids, Vol.40 , (1990), p. 345-360.

[65] T.W. Shield, P.H. Leo, and W. C.C. Grebner, Acta Mater., Vol. 45 , (1997) , p.67-74.

[66] J. A. Shaw , Int. J. Solids and Struct., Vol. 39 , (2002), p.1275-1305.

[67] O.P. Bruno, P.H. Leo, and F. Reitich Phys. Rev. Lett. 74 , (1995), p.746-749.

[68] J. A. Shaw, and S. Kyriakides, Int. J. Plasticity, Vol. 13 , (1998) ,p. 837-871.

[69]P.H. Leo, T.W. Shield, and O.P Bruno, Acta metal. Mater., Vol. 41 , (1993), p.2477-2485.

[70] M.F. Ashby, and D.R.H. Jones, Engineering Materials-2 , $2^{\text {nd }}$ edition, Butterworth Heinemann, (2001). 